\title{
Simultaneous HPLC-MS Determination of Loganin, Morroniside and Paeoniflorin in Rat Plasma; Pharmacokinetics of Liuwei Dihuang Pills
}

\author{
GUIYAN YUAN, R. ZHANG, XIAN LI, W. LI, RONG LI, B. WANG AND R. GUO* \\ Institute of Clinical Pharmacology, Qilu Hospital of Shandong University, Jinan, China
}

\begin{abstract}
Yuan et al.: HPLC-MS and Pharmacokinetics of Loganin, Morroniside and Paeoniflorin in Liuwei Dihuang Pills
\end{abstract}

\begin{abstract}
A novel high performance liquid chromatography-mass spectrometry method for simultaneous determination of loganin, morroniside and paeoniflorin in rat plasma was developed, validated and applied to pharmacokinetic study of Liuwei Dihuang pills. Samples were prepared by liquid-liquid extraction with ethyl acetate. Separation was performed on a Wondasil $C_{18}$ column $(150 \times 4.6 \mathrm{~mm}, 5 \mu \mathrm{m})$ using a mixture of methanol and $0.02 \%$ formic acid water solution $(28: 72, \mathrm{v} / \mathrm{v})$ as the mobile phase. The flow rate and column temperature were set at $0.7 \mathrm{ml} / \mathrm{min}$ and $30^{\circ}$. The compounds were detected in selected ion monitoring mode using an ESI source in the negative mode. The method was linear over the ranges of 5-1000 $\mathrm{ng} / \mathrm{ml}$ for the three analytes, with all determined correlation coefficients exceeding 0.993 . All of the lower limits of quantification of the three analytes were $5 \mathrm{ng} / \mathrm{ml}$. The intra-day and inter-day precisions were less than $7.50 \%$, and the accuracy ranged from -6.57 to $7.90 \%$. The mean recoveries of the analytes were higher than $68.86 \%$, and the matrix effects were between 92.35 and $103.54 \%$. This method was simple, sensitive and reliable, and could be used to monitor pharmacokinetics of loganin, morroniside and paeoniflorin. Moreover, this study might be helpful for quality control and guiding the clinical application of Liuwei Dihuang pills.
\end{abstract}

Key words: HPLC-MS, Liuwei Dihuang pill, loganin, morroniside, paeoniflorin, pharmacokinetics

Traditional Chinese medicine (TCM) and its preparations have been used in China for thousands of years and have become increasingly popular around the world in recent years because of their special efficacy, low toxicity and fewer side effects ${ }^{[1,2]}$. In the practice of TCM there are few herbal drugs being used alone. Conversely, formulae consisting of several drugs is the dominant administration mode. The complexity of active components and the lack of conclusive drug mechanisms make it difficult to be recognized by other countries. Pharmacokinetic studies of major active ingredients provide a suitable way to well understand the mechanism of drug effects, which are of importance to evaluate and improve the efficacy and safety of TCM.

Liuwei Dihuang (LD) is a well-known classical traditional Chinese formula, which has been clinically used for the treatment of kidney-Yin deficiency syndrome under TCM prescriptive terms in China for a long time ${ }^{[3]}$, which includes symptoms of soreness of the loins and the limbs, dizziness, tinnitus, and sweating. The formulation consisted of six Chinese herbal ingredients, including Radix rehmannide preparata, Rhizoma Dioscoreae, Fructus corni, Cortex moutan, Rhizoma alismatis and Poria. According to recent pharmacological studies, LD is effective in maintaining neuroendocrine immunomodulation balance ${ }^{[4,5]}$, improving cognitive function ${ }^{[6,7]}$, and improving or restoring the symptoms associated with aging and geriatric diseases ${ }^{[8]}$. Phytochemical investigation has found that LD contains many constituents including glycosides, phenols, acids, tannins and so on. Among these, glycosides, such as loganin, morroniside and paeoniflorin, are considered as the major bioactive

This is an open access article distributed under the terms of the Creative Commons Attribution-NonCommercial-ShareAlike 3.0 License, which allows others to remix, tweak, and build upon the work non-commercially, as long as the author is credited and the new creations are licensed under the identical terms

Accepted 09 December 2018

Revised 02 May 2018

Received 13 November 2017

Indian J Pharm Sci 2019;81(1):129-137 
constituents most contributing to the pharmacological efficacy of LD. Therefore, for further development and rational use of $\mathrm{LD}$, it is important to develop a method for quantitative analysis of loganlin, morroniside and paeoniflorin in vivo and study the pharmacokinetics of LD.

Till date, several papers have described high performance liquid chromatography (HPLC) methods for determination and pharmacokinetic studies of loganin ${ }^{[9]}$, morroniside ${ }^{[10]}$ and paeoniflorin ${ }^{[11]}$ independently, but it is more important to simultaneously determine these bioactive components in biological samples as much as possible due to the complexity of ingredients in herbal medicines. A few articles reported HPLC ${ }^{[12]}$ or ultra-performance liquid chromatography-mass spectrometry (UPLCMS) ${ }^{[13]}$ methods for the simultaneous determination of loganin and morroniside in mouse or rat plasma after intravenous or oral administration of Cornus officinalis. However, there are no reports available on the simultaneous determination and pharmacokinetic study of loganin, morroniside and paeoniflorin in rat plasma after oral administration of LD pills. Therefore, in the present study, a sensitive, specific and accurate HPLC-MS method for the simultaneous determination of loganin, morroniside and paeoniflorin in rat plasma was established, validated and successfully applied to the pharmacokinetic study of LD pills in rats.

\section{MATERIALS AND METHODS}

LD pills produced by Tong Ren Tang Technologies Co. Ltd. (Lot number 7073615, Beijing, China), were purchased from Jianlian Traditional Chinese Medicine Store (Jinan, China). The reference standards of loganin (99.9\%, lot number 111640-200503), paeoniflorin (99.8\%, lot number 110736-200732) and bergenin (IS, $99.9 \%$, lot number111532-200202) were obtained from National Institute for the Control of Pharmaceutical and Biological Products (Beijing, China), while the reference standard of morroniside $(99.6 \%$, batch no: 20090106) was provided by Shandong Analysis and Test Center (Jinan, China). HPLC-grade methanol (lot number H02E73) and ethyl acetate (lot number HCQG2H) were obtained from JT Baker Company (USA) and Tedia Company Inc. (USA), respectively. Analytical grade formic acid (lot number T20060124) was provided by Sinopharm Chemical Reagent Co. Ltd (Shanghai, China). Ultrapure water was used for all analyses.

\section{Instrumentation and analytical conditions:}

Sample analysis was performed on an Agilent 1200 series HPLC system and Agilent 6410 Triple Quadrupole mass spectrometer equipped with an electrospray ionization source (Agilent technologies, USA). All data were processed with the MassHunter B_01_04 software. The separation was achieved on a Wondasil $\mathrm{C}_{18}$ column $(150 \times 4.6 \mathrm{~mm}, 5 \mu \mathrm{m}$, GL Sciences Inc., Japan) at $30^{\circ}$. The mobile phase was a mixture of methanol- $0.02 \%$ formic acid water solution $(28: 72, \mathrm{v} / \mathrm{v})$, which was pumped at a flow rate of $0.7 \mathrm{ml} / \mathrm{min}$. The injection volume was $10 \mu \mathrm{l}$. The ESI source was set to negative ion mode using the following parameters: capillary voltage $4000 \mathrm{~V}$, desolvation gas (nitrogen) heated at $350^{\circ}(9 \mathrm{l} / \mathrm{min})$, nebulizer pressure $50 \mathrm{psi}$. The fragment voltages were $110 \mathrm{~V}$ for loganin, morroniside and IS, and $100 \mathrm{~V}$ for paeoniflorin. The quantification was performed in the selected ion monitoring (SIM) mode at $\mathrm{m} / \mathrm{z} 435.2$ for loganin, $\mathrm{m} / \mathrm{z} 451.2$ for morroniside, $\mathrm{m} / \mathrm{z} 525.2$ for paeoniflorin, and $\mathrm{m} / \mathrm{z} 327.1$ for IS.

\section{Preparation of LD pills suspension for animal oral administration:}

LD pills were ground to a powder and dissolved in $0.4 \%$ carboxymethylcellulose sodium (CMC$\mathrm{Na})$ solution. The content of loganin, morroniside and paeoniflorin determined by a HPLC method ${ }^{[14]}$ developed and validated by our lab was $1.44,1.87$ and $0.99 \mathrm{mg} / \mathrm{g}$, respectively.

\section{Preparation of standard and quality control (QC) samples:}

Stock solutions were independently prepared by dissolving $10.02 \mathrm{mg}$ loganin, $10.04 \mathrm{mg}$ morroniside, $10.03 \mathrm{mg}$ paeoniflorin and $1.00 \mathrm{mg}$ bergenin (internal standard, IS) in $10 \mathrm{ml}$ methanol. A series of mixed standard working solutions (10000, 1000 and $100 \mathrm{ng} / \mathrm{ml}$ ) were prepared by appropriate dilution of their respective stock solutions with methanol. The IS working solution was prepared at a final concentration of $100 \mathrm{ng} / \mathrm{ml}$ in methanol.

Appropriate volumes of mixed standard working solutions were added to a $10 \mathrm{ml}$ plastic tube, and evaporated to dryness at $40^{\circ}$ under a gentle stream of nitrogen. Then $100 \mu \mathrm{l}$ of rat plasma was added to the tube to obtain the calibration standards. The concentrations of calibration curves of loganin, morroniside and paeoniflorin were all 5, 10, 50, 100, 250, 500 and $1000 \mathrm{ng} / \mathrm{ml}$. The QC samples were similarly prepared 
at low, medium and high concentrations of 10, 100 and $750 \mathrm{ng} / \mathrm{ml}$ for the three analytes. All the prepared solutions were kept at $4^{\circ}$ and brought to room temperature before use.

\section{Plasma sample preparation:}

One hundred microlitres of plasma was mixed with $10 \mu \mathrm{IS}$ solution $(100 \mathrm{ng} / \mathrm{ml})$ and $3.5 \mathrm{ml}$ ethyl acetate. The mixture was vortexed for $2 \mathrm{~min}$ and centrifuged at $5000 \mathrm{rpm}$ for $5 \mathrm{~min}$. The organic layer was transferred to another tube, and evaporated to dryness in a thermostatic water bath at $40^{\circ}$ under a gentle stream of nitrogen. The residue was reconstituted with $100 \mu \mathrm{l}$ methanol- $0.2 \%$ formic acid water solution $(28: 72, v / v)$. And $10 \mu l$ of the solution was injected for analysis.

\section{Method validation:}

The analytical method was validated for its selectivity, calibration curve, lower limit of quantification (LLOQ), accuracy, intra- and inter-day precision, extraction recovery, matrix effect and stability according to the Food and Drug Administration guidelines ${ }^{[15]}$. The selectivity of the method was investigated by analysing six individual blank rat plasma samples. The chromatographic findings of each blank plasma were compared with the spiked rat plasma containing loganin, morroniside, paeoniflorin or IS and rat plasma after oral administration of LD pills to check for interferences.

Calibration curves were prepared by assaying standard plasma samples at seven different concentrations. The linearity of each calibration curve was determined by plotting the peak area ratio (y) of the analyte to the IS against the nominal concentration $(x)$ of the analyte with weighted $\left(1 / \mathrm{x}^{2}\right)$ least squares linear regression. The LLOQ was determined at the lowest concentration point of the calibration curve. Its measured precision, expressed as the relative standard deviation (RSD \%), was within $\pm 20 \%$, and its accuracy, expressed as relative error (RE \%), was also within $\pm 20 \%$.

The intra-day precision and accuracy were estimated by determining five replicate QC samples at three different concentration (low, medium and high) levels on the same day. The inter-day precision and accuracy were assessed by analysing five replicate QC samples at three concentrations on three consecutive days using calibration curves obtained daily. The precision and accuracy respectively expressed by RSD and RE were required no more than $15 \%$ and $\pm 15 \%$ at each concentration level.

The extraction recovery and matrix effect of the three analytes were determined by analysing five replicates of the plasma samples at three QC concentrations. The extraction recoveries were evaluated by comparing the peak areas of the analytes spiked in post-extraction samples with those spiked in pre-extraction samples at the same concentration. The matrix effects were calculated by comparing the peak areas of the analytes obtained from post-extraction spiked plasma samples with those obtained from the pure standard solutions at the same concentration. The extraction recovery and matrix effect of IS were similarly evaluated at one concentration.

The stability of the three analytes in rat plasma was studied by analysing five replicate QC samples at three different concentrations under different storage conditions including: storage at room temperature for $6 \mathrm{~h}$ after extraction, storage at $-20^{\circ}$ for 1 day and $7 \mathrm{~d}$ and after 3 freeze/thaw cycles. All QC samples were determined by using a calibration curve plotted daily. The analytes were considered stable with the deviation from the nominal concentration within $\pm 15 \%$.

\section{Application to a pharmacokinetic study:}

Male Wistar rats weighing 280 to $320 \mathrm{~g}$ were obtained from the experimental Animal Center of Shandong University, animal certification number was SCXK(Lu)20090001. All animals were housed under standard conditions of temperature, humidity and light with food (laboratory rodent chow) and water provided ad libitum and were acclimated in the laboratory for at least one week prior to experiment. Before administration, the animals were fasted overnight with free access of water. Pooled drugfree plasma was obtained from healthy rats and used for preparing calibration curves and QC samples. All of the experiments were carried out according to the Guidelines for the Care and Use of Laboratory Animals, and were approved by the Animal Ethics Committee of Shandong University (Jinan, China).

Six rats were administered an oral dose of $10 \mathrm{~g} / \mathrm{kg}$ LD pills (containing loganin $14.4 \mathrm{mg} / \mathrm{kg}$, morroniside $18.7 \mathrm{mg} / \mathrm{kg}$ and paeoniflorin $9.9 \mathrm{mg} / \mathrm{kg}$ ) CMC$\mathrm{Na}$ suspension. Venous blood samples $(0.3 \mathrm{ml})$ were collected through jugular sinus in heparinized $1.5 \mathrm{ml}$ tubes before administration and at 0.083 , $0.167,0.25,0.5,0.75,1,1.5,2,3,4,6,8,12,24 \mathrm{~h}$ 
after administration, and then centrifuged at 5000 rpm for 5 min immediately. The plasma samples were withdrawn and stored at $-20^{\circ}$ for further analysis. The concentration-time data were analysed using Drug and Statistic 2.0 pharmacokinetic software (Chinese Pharmacokinetics Association, Anhui, China) to calculate the main pharmacokinetic parameters with the non-compartment model, such as the area under plasma concentration-time curve $\left(\mathrm{AUC}_{0-\mathrm{t}}\right)$, the area under plasma concentration-time curve to time infinity $\left(\mathrm{AUC}_{0-\infty}\right)$, maximum plasma concentration $\left(\mathrm{C}_{\max }\right)$, time to reach the maximum plasma concentration $\left(\mathrm{T}_{\max }\right)$ and elimination half-life $\left(\mathrm{t}_{1 / 2}\right) \cdot \mathrm{C}_{\max }$ and $\mathrm{T}_{\max }$ were obtained directly from the plasma concentration-time curve. Data were expressed in mean and standard deviation (SD).

\section{RESULTS AND DISCUSSION}

Initially, the previously developed HPLC method ${ }^{[14]}$ reported from our laboratory for determination four ingredients in LD pills was adopted to determine loganin, morroniside and paeoniflorin. But, at the end, HPLC-MS method was employed in this study since the sensitivity of HPLC instrument could not meet the analytical requirement to detect the low plasma concentration of the three analytes in rats. To obtain the best mass spectrometry conditions, the standard solutions of the three analytes and IS were separately infused into the mass spectrometer, and were studied in both positive and negative ion modes. The results showed that the response of the analytes was much stronger in negative ion mode than that in positive ion mode. Due to the instability and low ion response, no product ion of the analytes could be used for quantification. So the SIM mode and precursor ion were used for quantification because of the high response, which could satisfy the requirement of the detection. The selected precursor ions were as follows: $\mathrm{m} / \mathrm{z} 435.2$ for loganin, $\mathrm{m} / \mathrm{z} 451.2$ for morroniside, $\mathrm{m} / \mathrm{z} 525.2$ for paeoniflorin, and $\mathrm{m} / \mathrm{z} 327.1$ for IS. The full scan mass spectrometry with the information of fragmentation pattern is shown in fig. 1. The fragment voltages for each analyte were further optimized, which were $110 \mathrm{~V}$ for loganin, morroniside and IS, and $100 \mathrm{~V}$ for paeoniflorin.

Chromatographic conditions were optimized to improve the peak shape, increase the signal response of the analytes, and shorten the run time. The mobile phase systems of methanol-water and acetonitrilewater in different proportions were tested. The response of the analytes was obviously higher with methanolwater as the mobile phase than that with acetonitrilewater. Different buffers, including ammonium acetate and formic acid, were tested to determine the optimal mobile phase. The results indicated that the peak strengths of the analytes increased dramatically when formic acid was added to the mobile phase, while ammonium acetate could inhibit the response of the analytes in negative ion mode. Different concentrations of formic acid in mobile phase were investigated. It was demonstrated that $0.02 \%$ formic acid could make higher response and better peak shape. Therefore, methanol- $0.02 \%$ formic acid water was selected.

In order to minimize the matrix effect and increase the extraction recovery, liquid-liquid extraction (LLE) and protein precipitation (PPT) were compared for sample preparation. PPT method (methanol or acetonitrile as precipitant) was not selected because of its high MS noise level, while LLE could produce a clean and concentrated sample. Different organic extracting solvents, including acetone, chloroform and ethyl acetate, were used, and ethyl acetate could offer satisfactory extraction efficiency for the analytes and IS.

A suitable IS is crucial for biological sample analysis, controlling for the variability in the extraction, HPLC injection and ionization. Bergenin was chosen as the IS because it exhibited a good response, peak shape and appropriate retention time. And no significant direct interference was observed.

Six blank plasma samples respectively from six rats were used to investigate the selectivity of the method. Typical chromatograms obtained from blank plasma, spiked plasma sample with the analytes $(100 \mathrm{ng} / \mathrm{ml})$ and IS, and plasma sample collected after 45 min after oral administration of LD pills are shown in fig. 2. All the analyte and IS peaks were detected with excellent resolution and good peak shapes, and no interference from the endogenous substances was observed at the retention time of the analytes and the IS. The retention time of loganin, morroniside, paeoniflorin and IS was $16.5,8.4,18.6$ and $6.7 \mathrm{~min}$.

Calibration curves of the three analytes exhibited good linearity in the ranges of $5-1000 \mathrm{ng} / \mathrm{ml}$. The Eqns for loganin, morroniside and paeoniflorin were $\mathrm{y}=0.0171 \mathrm{x}+0.0026 \quad\left(\mathrm{r}^{2}=0.996\right), \quad \mathrm{y}=0.0072 \mathrm{x}+0.0044$ $\left(\mathrm{r}^{2}=0.998\right), \quad$ and $\quad \mathrm{y}=0.0095 \mathrm{x}-0.0122 \quad\left(\mathrm{r}^{2}=0.993\right)$, respectively. The LLOQs of loganin, morroniside and paeoniflorin were all $5 \mathrm{ng} / \mathrm{ml}$, with $\mathrm{RSD}$ and 

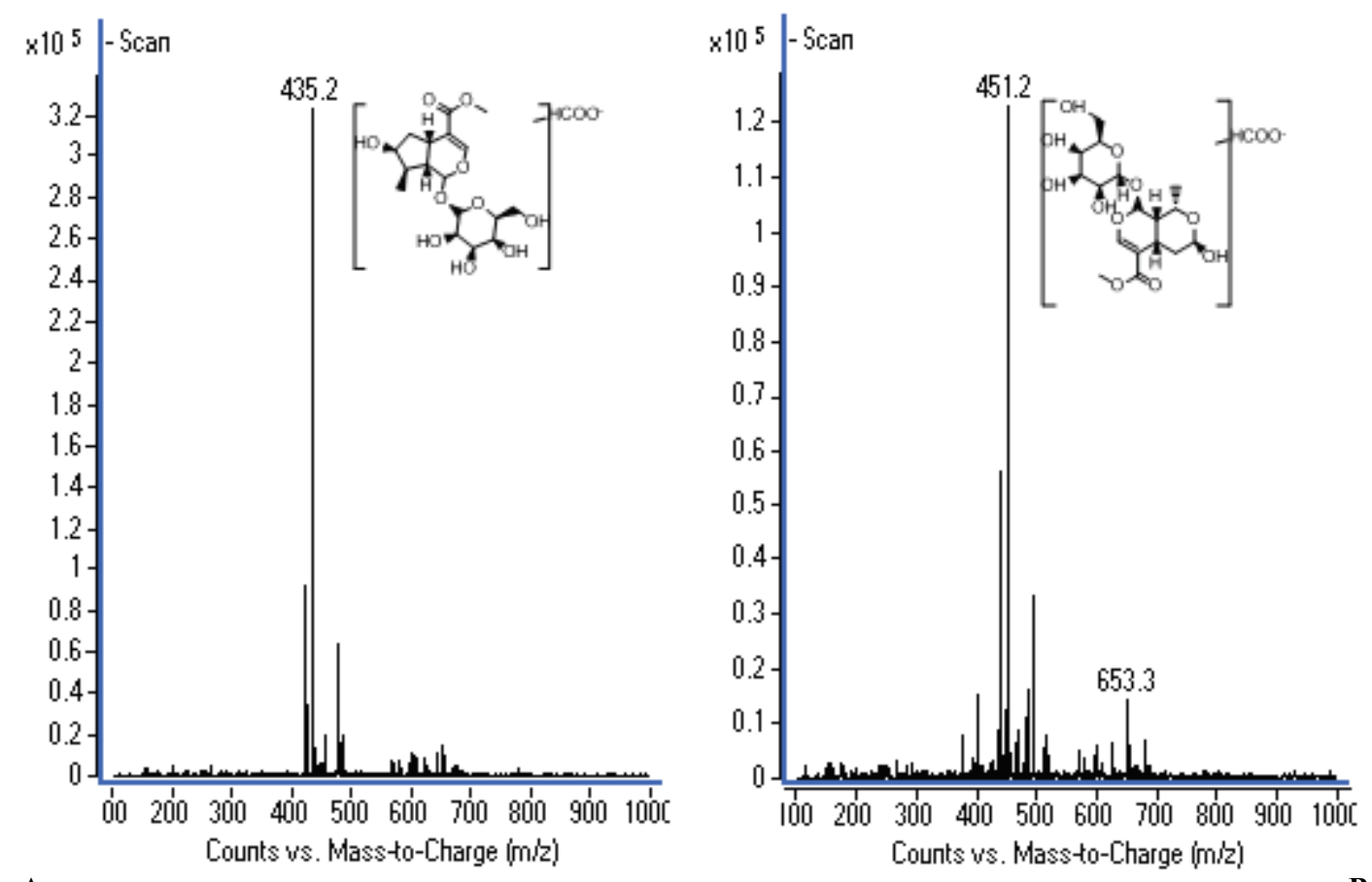

A
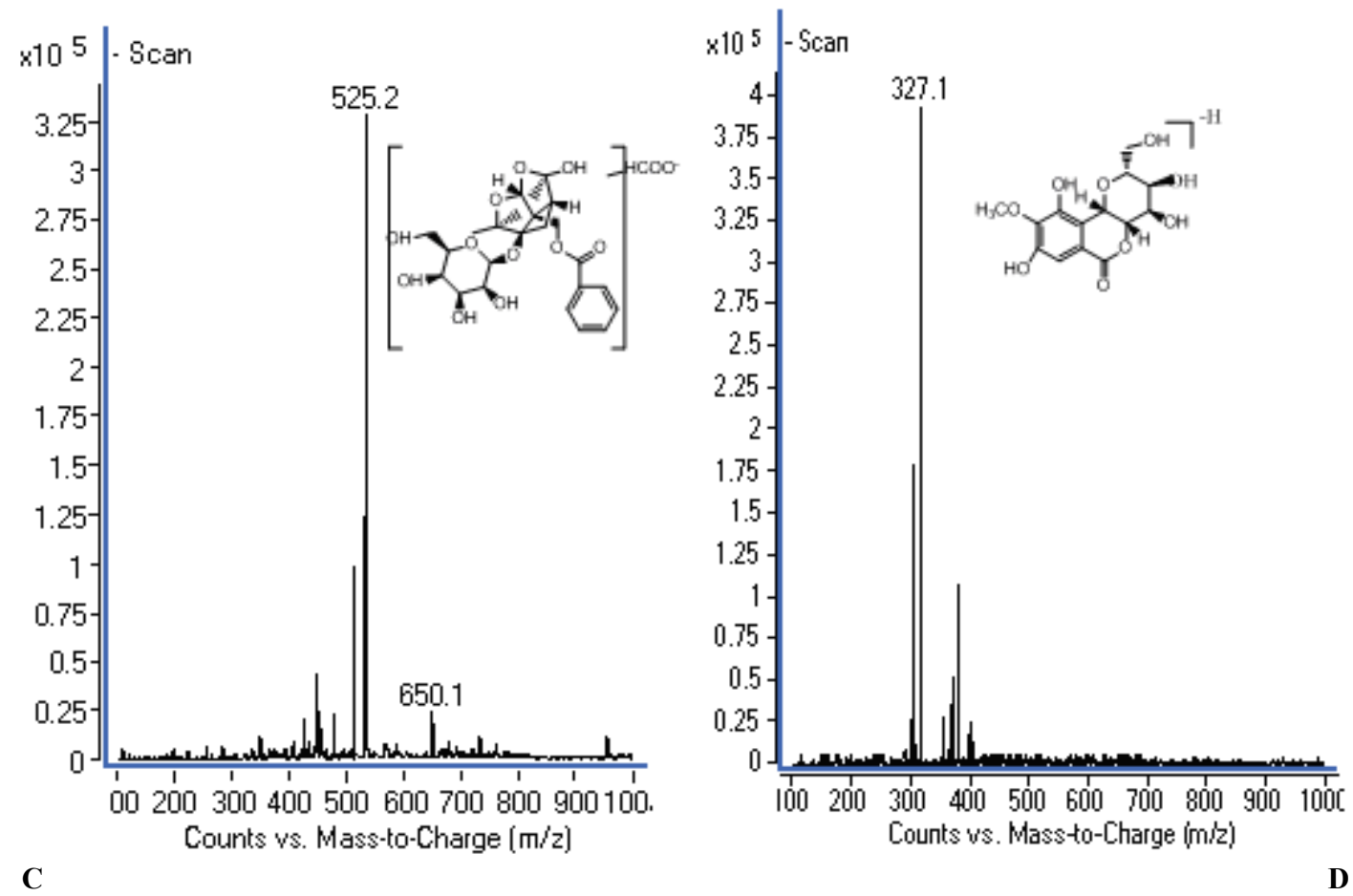

Fig. 1: Full scan mass spectrometry with fragmentation pattern Full scan mass spectrometry with information of fragmentation pattern of (A) loganin, (B) morroniside, (C) paeoniflorin and (D) bergenin

RE 1.817 and $1.02 \%, 1.215$ and $0.6 \%, 1.091$ and $0.42 \%$, respectively.

Precision and accuracy results are shown in Table 1. Five replicates of the QC samples were analyzed at 3 concentration levels of the analytes on the same day and on 3 different days. The intra-day precision (RSD) ranged from 0.033 to $7.50 \%$, and accuracy (RE) ranged between -6.57 and $3.11 \%$. Inter-day precision
(RSD) ranged from 0.031 to $6.39 \%$, and accuracy (RE) ranged between -2.35 and $7.90 \%$. Thus, this developed method was precise and accurate for satisfying the related rules of guidelines for biological sample analysis method.

The extraction solvent used in this method showed good extraction efficiency. The mean extraction recoveries of the three analytes in rat plasma at low, middle and 

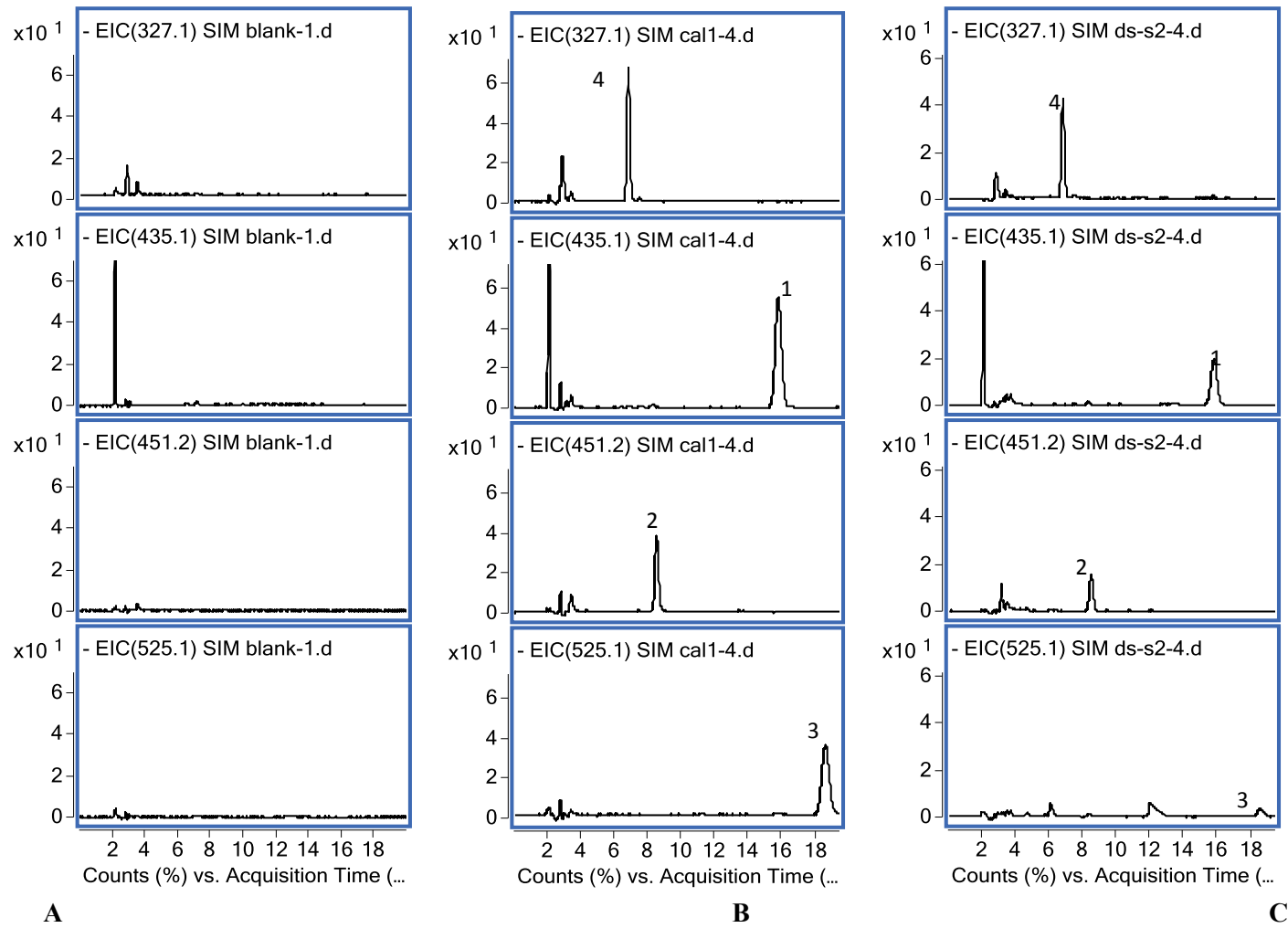

Fig. 2: Typical chromatograms

(A) Blank plasma, (B) spiked plasma with the analytes (1-loganin, 2-morroniside and 3-paeoniflorin) and IS (4-bergenin), and (C) plasma sample collected after $\mathbf{4 5}$ min after oral administration of LD pills

\section{TABLE 1: PRECISION AND ACCURACY OF THE THREE ANALYTES IN RAT PLASMA}

\begin{tabular}{lcccccc}
\hline Compounds & $\begin{array}{c}\text { Spiked } \\
\text { concentration } \\
(\mathbf{n g} / \mathbf{m l})\end{array}$ & $\begin{array}{c}\text { Measured } \\
\text { concentration } \\
(\text { Mean } \pm \text { SD }, \mathbf{n g} / \mathbf{m l})\end{array}$ & $\begin{array}{c}\text { Intra-day } \\
\text { precision } \\
(\%)\end{array}$ & $\begin{array}{c}\text { Intra-day } \\
\text { accuracy }(\%)\end{array}$ & $\begin{array}{c}\text { Inter-day } \\
\text { precision } \\
(\%)\end{array}$ & $\begin{array}{c}\text { Inter-day } \\
\text { accuracy }(\%)\end{array}$ \\
\hline \multirow{3}{*}{ Loganin } & 10 & $10.79 \pm 0.64$ & 5.32 & 3.07 & 5.88 & 7.90 \\
& 100 & $97.65 \pm 4.48$ & 3.27 & -4.41 & 4.59 & -2.35 \\
Morroniside & 750 & $770.40 \pm 23.52$ & 1.98 & 2.79 & 3.05 & 2.72 \\
& 10 & $9.94 \pm 0.56$ & 4.17 & -4.80 & 5.62 & -0.60 \\
& 100 & $98.06 \pm 5.24$ & 4.06 & -6.57 & 5.34 & -1.94 \\
Paeoniflorin & 750 & $772.85 \pm 28.08$ & 2.88 & 3.11 & 3.63 & 3.05 \\
& 10 & $10.50 \pm 0.61$ & 7.50 & 2.10 & 5.81 & 5.00 \\
& 100 & $103.21 \pm 6.60$ & 4.85 & 0.24 & 6.39 & 3.21 \\
\hline
\end{tabular}

high concentration were $76.91 \pm 3.77,78.40 \pm 3.18$, $79.17 \pm 5.95 \%$ for loganin, $79.89 \pm 9.06,68.86 \pm 2.60$, $73.04 \pm 6.92 \%$ for morroniside, and $78.52 \pm 9.86$, $82.77 \pm 2.66, \quad 82.91 \pm 7.48 \%$ for paeoniflorin, respectively, whereas $50.32 \pm 6.83 \%$ for IS. With regard to matrix effect, all the calculated values were between 92.35 and $103.54 \%$. No obvious matrix effect was observed, indicating that no co-eluting substance influenced the ionization of the analytes and IS. All of these results were in acceptable ranges.

Stability data of the 3 analytes are summarized in Table 2. The results indicated that the 3 analytes in rat plasma were stable at least for two freeze-thaw cycles or at $-20^{\circ}$ for $7 \mathrm{~d}$. In addition, the three analytes were also stable for $6 \mathrm{~h}$ after extraction at room temperature.

To the best of our knowledge, few studies have focused on the pharmacokinetics of LD pills, especially studies for its multi components. In this study, a validated HPLC-MS method was successfully applied to the pharmacokinetic studies of loganin and morroniside following oral administration of LD pills to male rats $(n=6)$, but to a lesser extent for determining pharmacokinetics of paeoniflorin, because most times the plasma levels of paeoniflorin in rat were below the LLOQ ( $5 \mathrm{ng} / \mathrm{ml})$, especially those samples from 2 to $24 \mathrm{~h}$ after administration. The following reasons might 


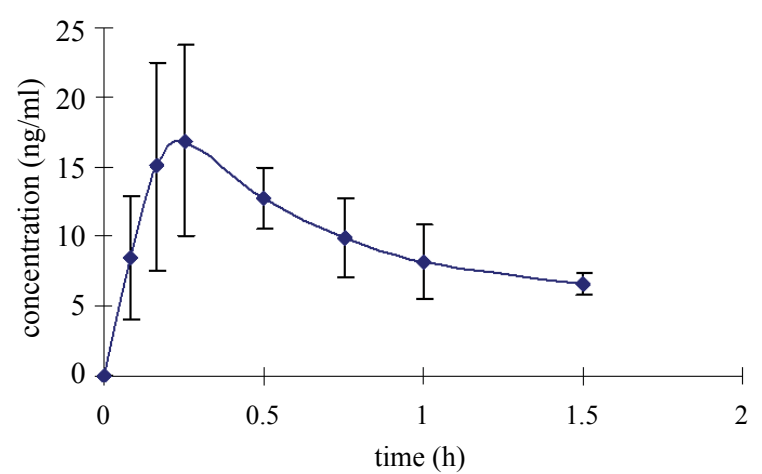

A

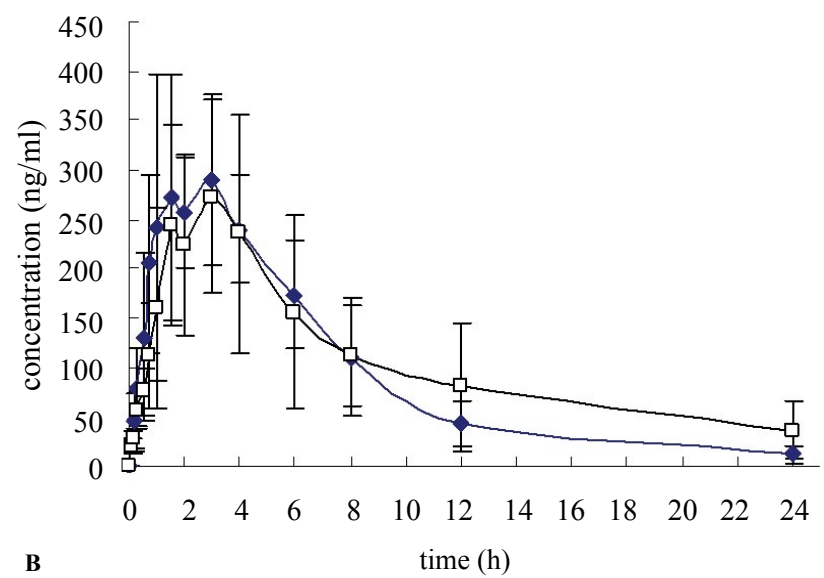

Fig. 3: Mean plasma concentration-time profiles of paeoniflorin, loganin and morroniside

Mean plasma concentration-time curves of (A) paeoniflorin, (B) loganin and morroniside in rats after oral administration LD pills (n=6). A: (—-) Paeoniflorin; B: (-४) loganin, (一- $\square$ morroniside

TABLE 2: THE STABILITY OF THE THREE ANALYTES IN RAT PLASMA

\begin{tabular}{lcccc}
\hline \multirow{2}{*}{$\begin{array}{c}\text { Spiked } \\
\text { Compounds concentration } \\
(\mathrm{ng} / \mathrm{ml})\end{array}$} & $\begin{array}{c}\text { Two } \\
\text { freeze- } \\
\text { thaw } \\
\text { cycles }\end{array}$ & $\begin{array}{c}\text { At }-20^{\circ} \\
\text { for } 7 \\
\text { days }\end{array}$ & $\begin{array}{c}\text { 6 h h after } \\
\text { extraction }\end{array}$ \\
\hline \multirow{3}{*}{ Loganin } & 10 & -2.17 & -1.32 & -5.03 \\
& 100 & -0.37 & -1.81 & -2.70 \\
& 750 & -0.49 & -0.63 & -0.06 \\
Morroniside & 10 & -3.71 & -0.79 & -3.71 \\
& 100 & -2.55 & -0.93 & -3.21 \\
& 750 & 0.42 & -0.36 & 0.10 \\
Paeoniflorin & 10 & 2.25 & 1.49 & 2.14 \\
& 100 & -1.52 & 3.49 & -1.48 \\
& 750 & 0.75 & 0.26 & 0.16 \\
\hline
\end{tabular}

explain these results. Firstly the lower administered dosage of LD pills $(10 \mathrm{~g} / \mathrm{kg}$ containing $9.9 \mathrm{mg} / \mathrm{kg}$ paeoniflorin) compared to that reported in published pharmacokinetic studies of paeoniflorin, where doses of paeoniflorin employed was $300 \mathrm{mg} / \mathrm{kg}\left(\mathrm{C}_{\max } 3340 \pm\right.$ $1180 \mathrm{ng} / \mathrm{ml})^{[16]}, 294.78 \mathrm{mg} / \mathrm{kg}(1550 \pm 530 \mathrm{ng} / \mathrm{ml})^{[17]}$ or $88 \mathrm{mg} / \mathrm{kg}(870 \pm 240 \mathrm{ng} / \mathrm{ml})^{[18]}$. The other reason might be the drug-drug interaction. Other ingredients existing in LD pills may enhance the metabolic transformation or inhibit the absorption of paeoniflorin, then reduce extremely the concentration of paeoniflorin in rat plasma $^{[19,20]}$. But anyway, paeoniflorin can be detected in rat plasma during 0.083-1.5 h after administration. The mean concentration-time curve of paeoniflorin could also show some pharmacokinetic characteristics (fig. 3A). The pharmacokinetic parameters calculated using the detected concentration data are shown in Table 3. It might not be very accurate due to low concentrations detected. But it could still provide some experimental references for other researchers. In addition, the pharmacokinetics of paeoniflorin after oral administration of high dosage of LD pills is also needed to be further investigated.

The profiles of the mean plasma concentration versus time of loganin and morroniside are illustrated in fig. 3B. The main pharmacokinetic parameters calculated using a noncompartmental model are presented in Table 3 . The results showed that loganin and morroniside have similar oral absorption behaviours, which might be caused by similar structures. Loganin and morroniside were detected in rat plasma at $5 \mathrm{~min}$ after oral administration of LD pills, which indicated loganin and morroniside were absorbed quickly. A double-peak phenomenon was observed for loganin and morroniside after oral administration of LD pills, when the first peak occurred at around $1.33 \mathrm{~h}$ and the second at around $4 \mathrm{~h}$. These results were different from those observed in the study of pure loganin ${ }^{[21]}$ or pure morroniside ${ }^{[22]}$ administration at a dose of $20 \mathrm{mg} / \mathrm{kg}$, in which unimodality was observed for loganin or morroniside. The double-peak phenomenon might be due to the transformation from other similar ingredients in LD pills ${ }^{[23]}$. Because there were a large number of components with the same parent nucleus in LD pills, which could be easily converted to each other. The transformation between components could cause the concentration of a component to rise again and produce double-peak. The half-life of loganin and morroniside in this study was $4.40 \pm 1.63 \mathrm{~h}$ and $7.71 \pm 5.76 \mathrm{~h}$, respectively, which was longer than the study of pure loganin $(93.6 \pm 36.7 \mathrm{~min})$ or pure morroniside $(0.7 \pm 0.3 \mathrm{~h})$ administration. The $\mathrm{AUC}_{0 \text {-t }}$ of morroniside $(2622.37 \pm 864.17 \mathrm{ng} \cdot \mathrm{h} / \mathrm{ml})$ was higher

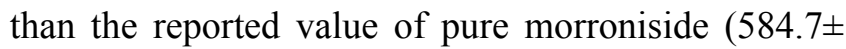
$290.9 \mathrm{ng} \cdot \mathrm{h} / \mathrm{ml}$ ). These results indicated that co-existing ingredients in LD pills could enhance the absorption of morroniside and delay the elimination of loganin and 
TABLE 3: PHARMACOKINETIC PARAMETERS OF PAEONIFLORIN, LOGANIN AND MORRONISIDE IN RATS

\begin{tabular}{lccc}
\hline Parameters & Loganin & Morroniside & Paeoniflorin \\
\hline $\mathrm{C}_{\max 1}(\mathrm{ng} / \mathrm{ml})$ & $295.54 \pm 123.57$ & $232.59 \pm 142.37$ & $19.26 \pm 7.62$ \\
$\mathrm{C}_{\max 2}(\mathrm{ng} / \mathrm{ml})$ & $287.17 \pm 90.15$ & $306.83 \pm 115.68$ & - \\
$\mathrm{T}_{\max 1}(\mathrm{~h})$ & $1.33 \pm 0.86$ & $1.33 \pm 0.49$ & $0.21 \pm 0.05$ \\
$\mathrm{~T}_{\max 2}(\mathrm{~h})$ & $4.17 \pm 1.94$ & $4.67 \pm 1.97$ & - \\
$\mathrm{t}_{1 / 2}(\mathrm{~h})$ & $4.40 \pm 1.63$ & $7.71 \pm 5.76$ & $1.46 \pm 1.30$ \\
$\mathrm{AUC}_{0-24}(\mathrm{ng} \cdot \mathrm{h} / \mathrm{ml})$ & $2275.17 \pm 553.83$ & $2622.37 \pm 864.17$ & $15.18 \pm 2.76$ \\
$\mathrm{AUC}_{0-\infty}(\mathrm{ng} \cdot \mathrm{h} / \mathrm{ml})$ & $2343.48 \pm 601.31$ & $3191.67 \pm 1531.92$ & $29.76 \pm 16.38$
\end{tabular}

Pharmacokinetic parameters of paeoniflorin, loganin and morroniside in rats after oral administration of LD pills, mean $\pm S D(n=6)$

morroniside. But the $\mathrm{AUC}_{0-\mathrm{t}}$ of loganin was lower than the reported value, the reason for which needs to be further clarified.

\section{Conflicts of interest:}

There is no conflict of interest.

\section{Financial assistance and scholarship:}

Nil.

\section{REFERENCES}

1. Williamson EM, Lorenc A, Booker A, Robinson N. The rise of traditional Chinese medicine and its Materia Medica: A comparison of frequency and safety of materials and species used in Europe and China. J Ethnopharmacol 2013;149(2):453-62.

2. Zhao M, Zhou Q, Ma W, Wei D. Exploring the ligand-protein networks in traditional Chinese medicine: current databases, methods, and applications. Evid Based Complement Alternat Med 2013;2013:806072.

3. Wang Y, Xu A. Zheng: A systems biology approach to diagnosis and treatments. Science 2014;346(6216):S13-S5.

4. Wang J, Yao K, Yang X, Liu W, Feng B, Ma J, et al. Chinese patent medicine Liu Wei Di Huang Wan combined with antihypertensive drugs, a new integrative medicine therapy, for the treatment of essential hypertension: A systematic review of randomized controlled trials. Evid Based Complement Alternat Med 2012;2012:714805.

5. He H, Yang X, Zeng X, Shi M, Yang J, Wu L, et al. Protective effect of Liuwei Dihuang decoction on early diabetic nephropathy induced by streptozotocin via modulating ETROS axis and matrix metalloproteinase activity in rats. $\mathrm{J}$ Pharm Pharmacol 2007;59(9):1297-305.

6. Huang Y, Zhang H, Yang S, Qiao H, Zhou W, Zhang Y. Liuwei Dihuang decoction facilitates the induction of long-term potentiation (LTP) in senescence accelerated mouse/prone 8 (SAMP8) hippocampal slices by inhibiting voltage-dependent calcium channels (VDCCs) and promoting N-methyl-daspartate receptor (NMDA) receptors. J Ethnopharmacol 2012;140(2):384-90.

7. Liu J, Feng L, Zhang M, Ma D, Wang S, Gu J, et al. Neuroprotective effect of Liuwei Dihuang decoction on cognition deficits of diabetic encephalopathy in streptozotocininduced diabetic rat. J Ethnopharmacol 2013;150(1):371-81.

8. Sangha JS, Sun X, Wally OS, Zhang K, Ji X, Wang Z, et al.
Liuwei Dihuang (LWDH), a traditional Chinese medicinal formula, protects against $\beta$-amyloid toxicity in transgenic Caenorhabditis elegans. PloS One 2012;7(8):e43990.

9. Xie YS, Zhang ZQ, Ruan JX. RP-HPLC determination of loganin in mouse plasma and its pharmacokinetics. Yao Xue Xue Bao 2002;37(7):548-50.

10. Li X, Wang Q, Zhang X, Sheng X, Zhou Y, Li M, et al. HPLC study of pharmacokinetics and tissue distribution of morroniside in rats. J Pharm Biomed Anal 2007;45(2):349-55.

11. Dai B, Liu L, Xiao Z, Leng W, Zou S. Determination of paeoniflorin in normal and yin asthenia rat serum after oral administration of Liuwei Dihuang pills. Chin J Tradit Med Sci Technol 2008;15(1):46-7.

12. Zhang LT, Ren LM, Wun JK. Studies on pharmacokinetics of loganin and morroniside in Cornus officinalis injection in mice. Zhongguo Zhong Yao Za Zhi 2003;28(6):509-12.

13. Zhao M, Tao J, Qian D, Liu P, Shang E, Jiang S, et al. Simultaneous determination of loganin, morroniside, catalpol and acteoside in normal and chronic kidney disease rat plasma by UPLC-MS for investigating the pharmacokinetics of Rehmannia glutinosa and Cornus officinalis Sieb drug pair extract. J Chromatogr B Analyt Technol Biomed Life Sci 2016;1009-1010:122-29.

14. Li X, Wang B, Yuan G, Wei C, Guo R. Determination of four effective constituents in Liuwei Dihuang pills by HPLC. Chin J Pharm 2010;41(2):126-28.

15. FDA, Food and Drug Administration. Guidance for Industry: Bioanalytical Method Validation, 2013. Available from: http:/www.fda.gov/downloads/drugs/ guidancecomplianceregulatoryinformation/guidances/ ucm368107.pdf.

16. Wang $\mathrm{CH}$, Wang $\mathrm{R}$, Cheng XM, He YQ, Wang ZT, Wu C, et al. Comparative pharmacokinetic study of paeoniflorin after oral administration of decoction of Radix Paeoniae Rubra and Radix Paeoniae Alba in rats. J Ethnopharmacol 2008;117:467-72.

17. Wu J, Yao N, Wang D. Determination of paeoniflorin in rat plasma by HPLC-MS/MS and its pharmacokinetics. China J Chin Mater Med 2008;33(20):2369-73.

18. Wen J, Qiao Y, Yang J, Liu X, Song Y, Liu Z, et al. UPLCMS/MS determination of paeoniflorin, naringin, naringenin and glycyrrhetinic acid in rat plasma and its application to a pharmacokinetic study after oral administration of Si-Ni-San decoction. J Pharm Biomed Anal 2012;66:271-7.

19. Guo C, Zhang L, Jiang H. Intragastric administration of Radix Paeoniae Rubra extracts versus Taohong Siwu Tang for the serum concentration of peoniflorin in mice. Chin J Clin Rehabil 2006;10(47):206-08. 
20. Liu D, Zhang L, Chen T. Pharmacokinetics study on paeoniflorin in Sini San. Chin J Exp Tradit Med Form 2005;11(2):36-8.

21. Li X, Wang Q, Zhang L, Yin W, Xu L, Li M. Pharmacokinetic study on loganin from Cornus officinalis Sieb. et Zucc. in rats. Chin J Pharm Anal 2007;27(1):4-7.

22. Xiong S, Li JL, Zhu XQ, Wang XY, Lv GY, Zhang
ZQ. Determination of morroniside concentration in rat plasma by high performance liquid chromatographytandem mass spectrometry. Zhongguo Zhong Yao Za Zhi 2013;38(19):3378-81.

23. Godfrey KR, Arundel PA, Dong Z, Bryant R. Modelling the double peak phenomenon in pharmacokinetics. Comput Methods Programs Biomed 2011;104(2):62-9. 